\title{
Efectos del programa "Adultos mayores activos y saludables" sobre los niveles de hemoglobina glicosilada y estado antropométrico en pacientes con DM2 mayores de 59 años usuarios del Centro Médico ESSalud Ayaviri, 2018
}

\begin{abstract}
Effects of the program "Active and healthy older adults" on glycosylated hemoglobin levels and anthropometric status in patients with DM2 older than 59 years users of the ESSalud Ayaviri Medical Center, 2018
\end{abstract}

Patthy Milagros Condori Choquepata1, Tirsa Abigail Mauricio Navarro²

\begin{abstract}
RESUMEN
Objetivo: determinar el efecto del programa "adultos mayores activos y saludables" sobre los niveles de hbA1c y estado antropométrico en pacientes con DM2 mayores de 59 años usuarios del centro Médico ESSalud Ayaviri - 2018. Metodología: estudio de diseño cuasi experimental, de corte longitudinal ya que se formó dos grupos de estudio con medidas antes y después del programa, una muestra de 113 adultos mayores quedando 91 participantes después de que se aplicaron los test de selección, siendo 51 el grupo de estudio y 41 el grupo control. El programa estuvo constituido por ejercicios combinados (aeróbicos y anaeróbicos) alternados de 15" cada uno durante 45' en 5 días por un periodo de 12 semanas, más una charla de alimentación saludable para el grupo control. Resultados: antes de la aplicación del programa se calculó el nivel de actividad física de la población, siendo esta un $60.4 \%$ de actividad baja seguido de un $39.6 \%$ de actividad moderada. Después de la aplicación del programa se observó que no existía diferencia significativa entre 1era y 2da medición de $\mathrm{HbA} 1 \mathrm{c}$ en pacientes adultos mayores con DM2 $(p>.05)$, así mismo hubo una diferencia clínica en los valores porcentuales de $0.2 \%-1 \%$, en cuanto al IMC hubo una diferencia estadísticamente significativa entre la primera y la segunda medición $(p<.05)$, en cuanto al ICC se obtuvo una diferencia significativa en la disminución de sus valores durante el segundo control al igual que con el test MNA $(p<.05)$. Conclusión: a nivel de significancia no hubo cambios en los valores de $\mathrm{HbA} 1 \mathrm{c}$ después del programa de ejercicios, pero sí cambios clínicos porcentuales. Por otro lado, si hubo efecto del programa en los cambios antropométricos de los pacientes, finalmente no hubo efecto a través del ICC sobre los niveles de HbA1c.
\end{abstract}

Palabras claves: Diabetes mellitus 2; ejercicios combinados; hemoglobina glicosilada; adultos mayores; Índice de masa corporal; índice de cintura cadera.

\section{ABSTRACT}

Objective: to determine the effect of the program "active and healthy older adults" on the levels of $\mathrm{hbA} 1 \mathrm{c}$ and anthropometric status in patients with DM2 older than 59 years users of the ESSalud Ayaviri Medical Center - 2018. Methodology: quasi-experimental design study, longitudinal since two study groups were formed with measures before and after the program, a sample of 113 older adults leaving 91 participants after the selection tests were applied, with 51 being the study group and 41 the control group. The program consisted of combined exercises (aerobic and anaerobic) alternating 15 "each for 45 'in 5 days for a period of 12 weeks, plus a healthy eating talk for the control group. Results: before the application of the program, the physical activity level of the population was calculated, this being $60.4 \%$ of low activity followed by $39.6 \%$ of moderate activity. After the application of the program it was observed that there was no significant difference between 1st and 2nd measurement of $\mathrm{HbA} 1 \mathrm{c}$

${ }^{1}$ Nutricionista en Clínica de Dios, Puerto Maldonado, Perú.

${ }^{2}$ Nutricionista en Aldea Infantil San Juan, Lima, Perú. 
in elderly patients with DM2 ( $p>.05)$, likewise there was a clinical difference in the percentage values of $0.2 \%-1 \%$, Regarding the BMI, there was a statistically significant difference between the first and second measurements $(p<.05)$, as for the ICC a significant difference was obtained in the decrease of its values during the second control as with the MNA test $(p<.05)$. Conclusion: at the level of significance there were no changes in the $\mathrm{HbA1c}$ values after the exercise program, but there were clinical percentage changes. On the other hand, if there was effect of the program on the patients' atropometric changes, there was finally no effect through the ICC on HbA1c levels.

Keywords: Cardiovascular risk, lifestyle, correlation.

\section{INTRODUCCIÓN}

Las enfermedades crónicas no transmisibles están teniendo una prevalencia incrementada en el mundo, entre ellas la Diabetes mellitus (DM), en consecuencia, la Federación internacional de Diabetes (2017), ha señalado que existen 415 millones de personas con DM, siendo la mayoría de los casos Diabetes Mellitus tipo 2 (DM2) (IDF, 2017). Asimismo, la Organización Mundial de la Salud en el 2016 proyectó que la DM será la 7 ma causa de mortalidad a nivel mundial para el 2030 (Villena, 2016). Así pues, la IDF en el 2013 propuso que los factores influyentes para el aumento de la DM entre ellos: sistema de salud con bajo rendimiento, baja conciencia entre el público general y profesionales de la salud, el lento inicio de los síntomas o la progresión de la DM2, ya que esta puede mantenerse sin detectar durante muchos años y pueden desarrollarse complicaciones (Fernandes,et al , 2014). En Perú la DM afecta al $7 \%$ de la población adulta, representada por el $96.8 \%$ de los casos, también es la primera causa de enfermedad renal crónica (ERC), como también de amputaciones no traumáticas de miembros inferiores y la sexta causa de ceguera (Villena, 2016).

En el primer semestre del 2017, en la Red de Salud Puno, se tiene registrado un total de 130 casos de DM, como también 740 de Hipertensión Arterial (HTA) y 1,730 de obesidad. Las cifras nos muestran que este grupo de enfermedades no transmisibles van aumentando de forma inquietante en relación al 2016, cuyas cifras anuales eran de 227 casos de DM, 1312 de HTA y 2,670 de obesidad y se considera que las personas con mayor repercusión a DM son mayores de 30 años (Paredes, 2017).

En el 2015 se calculó 5.0 millones de muertes atribuibles a la DM, y el grupo de población con la mayor proporción de muertes, fue fueron mujeres de 50 - 59 años que representan el $20 \%$ de todas las causas de mortalidad en ese grupo (Ministerio De Salud, 2016). Haciendo un comparativo de las muertes por DM en el 2014, que fueron 4.9 millones y las del 2015 que fue de 5.0 millones, nos podemos dar cuenta que, en vez de mejorar la prevalencia de esta enfermedad, se está volviendo un problema inquietante (IDF, 2016).

Por otra parte, se observa un incremento en la proporción de adultos mayores en la población peruana, siendo de $5,7 \%$ en el año 1950 a 9.9 \% en el año 2016 (Mamani, 2016). Asimismo, la IDF estima una prevalencia de DM en adultos de 20-79 años es de $6.1 \%$ equivalente a 1.143 , 600 millones de personas, de las cuales 317,700 no han sido diagnosticadas. Según la Encuesta Demográfica y de Salud Familiar (ENDES) realizada el 2014 sobre una muestra nacional en mayores de 15 años, se halló una prevalencia de $3.2 \%$ en DM diagnosticada; de la cual el $3.6 \%$ correspondía a las mujeres y $2.9 \%$ en varones. En un estudio realizado en Perú se encontró que la prevalencia de DM fue mayor en adultos mayores $(10.6 \%)$ y en personas sin alfabetización (18.8\%); según el mismo, la prevalencia de DM2 es mayor en la ciudad de Lima y la región costera cuya población tiene un estilo de vida occidentalizada, que en las regiones de la sierra y selva quienes consumen una dieta autóctona, menor consumo de comidas industrializadas y realizan mayor actividad física. En cuanto a la prevalencia de morbimortalidad, hay 6,853 admisiones hospitalarias por DM; 2,449 más que en el 2005, observándose un incremento de $55.6 \%$. En su mayoría provenientes de la costa $(59 \%)$ y adultos mayores de 60 años (46.9\%). Referente al tipo de DM, la mayoría de las hospitalizaciones se dan por DM2, representando el $94.2 \%$ de hospitalizaciones, DM1 $3 \%$ y 0,2 en DG. Las causas frecuentes de hospitalización 
Efectos del programa "Adultos mayores activos y saludables" sobre los niveles de hemoglobina glicosilada y estado antropométrico en pacientes con DM2 mayores de 59 años usuarios del Centro Médico ESSalud Ayaviri, 2018

en adultos con DM se dividen en tres grupos: las enfermedades infecciosas $(24.1 \%-29.8 \%)$, dentro de ellas pie diabético, infección urinaria y respiratoria destacándose como la causa de más mortalidad (34\% $-57.5 \%)$. La segunda causa es las emergencias diabéticas $(14.7 \%-24.1 \%)$, dentro de ellas se encuentran la hipoglicemia y cetoacidosis, mayormente en DM2 y por último, las enfermedades cardiovasculares $(6.6 \%$ $13.7 \%)$.

En una encuesta de 605 personas realizada el 2016 a pacientes diabéticos y familiares reveló que el $33 \%$ de las personas consideraba el acceso al tratamiento y educación diabetológica adecuado en el Perú, 32\% indicaron que el apoyo de la comunidad era apropiado y $27 \%$ dijeron que la calidad de los centros de salud encargados de su cuidado era buena, el $56 \%$ no sabía si había presupuesto designado para el control de esta enfermedad (Villena, 2016).

Por otra parte, el sedentarismo es una de las causas de mortalidad en personas diagnosticadas y no diagnosticadas con $\mathrm{M} 2$, provocando el $6 \%$ de todas las muertes, como también gracias a que la inactividad física va en aumento en muchos países, tanto que las personas con un nivel bajo de actividad tienen entre el $20 \%$ y $30 \%$ más riesgo de muerte que los que realizan al menos 30 minutos de actividad física moderada. Por consiguiente, es la causa principal de alrededor $21-25 \%$ de cánceres de mama y colon, $27 \%$ de casos de diabetes y $30 \%$ de cardiopatías isquémicas. Según ENDES (2012), los habitantes peruanos de 40 años a más realiza actividades sentado representando el $19.2 \%$, el $29.1 \%$ realiza esfuerzo leve para actividades de la vida diaria, $45.2 \%$ no realiza ningún deporte. Estas cifras se ven acentuadas en personas mayores de 60 años y en zonas urbanas (Gonzáles, 2016).

Así pues, la DM está acrecentando en nuestro país, ya que la mitad de los habitantes adultos y la cuarta parte de los habitantes niños presentan sobre peso y obesidad, dejándonos un futuro no muy asegurado, y si no contamos con un buen programa de prevención y control de dicha enfermedad, pues estaremos reafirmando las estadísticas de este aumento (Seclén, 2015).

En definitiva, la implementación de un programa de ejercicios en esta población es crucial para poder mantener un estilo de vida saludable y mejorar los síntomas que puede causar la DM2.

\section{METODOLOGÍA}

El estudio es de diseño cuasi experimental, longitudinal, porque se formó dos grupos de estudio, a uno se le aplicó el programa y el otro fue el control, con medidas antes y después de la aplicación del programa. El estudio se realizó en la ciudad de Ayaviri-Puno y la población estuvo conformada por 113 pacientes adultos mayores de más de 59 años con DM2 del Hospital III Juliaca-Centro Médico EsSalud Ayaviri, durante los meses de diciembre del 2017 a noviembre del 2018.

Se realizó muestreo no probabilístico por conveniencia y se aplicaron criterios de inclusión y exclusión, siendo 92 los participantes seleccionados.

\section{Instrumentos}

Se aplicaron tres pruebas: la prueba de equilibrio (Balance test); 30 segundos silla y de pie (The 30 second chair stand test) y la prueba de curvatura de brazo (arm curl test); los que aprobaron fueron entrevistados con el cuestionario internacional de actividad física (IPAQ) y Mini examen del estado nutricional (MNA).

\section{Método experimental}

Se dividió a la población en dos grupos de manera aleatoria: grupo de estudio (GE) con 51 participantes y el grupo control (GC) con 41 , se elaboró un programa de ejercicios combinados (anaeróbicos y aeróbicos) alternados de 15" cada uno, con una duración de 45 minutos cada sesión, 5 veces/semana por un periodo de 12 semanas (anexo 6); ejercicios que fueron aplicados solo al GE. Finalmente, para las pruebas de laboratorio se utilizó 1 kit de hemoglobina glicosilada x 100ml.

\section{Análisis de datos}

Se procedió al recojo de datos al inicio y al término del programa de ejercicios. El registro y ordenamiento de los datos obtenidos de las encuestas y del examen de laboratorio se realizaron con el programa Microsoft Excel 2010, el análisis de datos fue medido por el programa SPSS versión 23 y se utilizó la prueba de Wilcoxon de acuerdo al tipo de variable cuantitativa sin distribución normal. 


\section{RESULTADOS}

En la Tabla 1 se evidencia que el $52.9 \%$ de los participantes del grupo de estudio es de sexo femenino, el $78.4 \%$ tiene una edad que oscila entre 59 a 63 años. En el grupo control se observa que el $61 \%$ es de sexo masculino y el $75,6 \%$ tiene una edad que oscila entre 59 a 63 años.

Tabla 1

Distribución de la muestra según variables sociodemográficas.

\begin{tabular}{llll}
\multicolumn{1}{c}{ Grupo } & & & N \\
Grupo de estudio & Sexo & & \\
& Femenino & 27 & 52.9 \\
& Masculino & 24 & 47.1 \\
& Total & 51 & 100.0 \\
& 59 a 63 años & 40 & 78.4 \\
& 64 a 67 años & 11 & 21.6 \\
Total & 51 & 100.0 \\
& Sexo & & \\
& Femenino & 16 & 39.0 \\
& Masculino & 25 & 61.0 \\
& Edad & & 75.6 \\
& 59 a 63 años & 31 & 24.4 \\
& 64 a 67 años & 10 & 100.0 \\
\hline
\end{tabular}

En la tabla 2 se observa que no existe diferencia estadísticamente significativa entre la primera y la segunda medición de la HbA1c ( $p>0.5$ ) en el GE y el GC. Así mismo, desde el punto de vista clínico hubo una reducción del porcentaje de $\mathrm{HbA} 1 \mathrm{c}$ de $0.2 \%$ a $1 \%$.

Tabla 2

Análisis y comparación de la HbA1c antes y después de la intervención.

\begin{tabular}{|c|c|c|c|c|c|c|}
\hline \multirow[t]{2}{*}{ Grupo } & \multirow[t]{2}{*}{ HbA1c } & \multicolumn{2}{|c|}{ Pre test } & \multicolumn{2}{|c|}{ Pos test } & \multirow[t]{2}{*}{$\mathbf{p}^{*}$} \\
\hline & & $\mathbf{N}$ & $\%$ & $\mathbf{n}$ & $\%$ & \\
\hline \multirow[t]{4}{*}{ Grupo de estudio } & Controlado & 0 & 0 & 0 & 0 & 0.219 \\
\hline & $\begin{array}{l}\text { Control } \\
\text { suficiente }\end{array}$ & 41 & 80.4 & 45 & 88.2 & \\
\hline & Mal controlado & 10 & 19.6 & 6 & 11.8 & \\
\hline & Total & 51 & 100.0 & 51 & 100.0 & \\
\hline \multirow[t]{4}{*}{ Grupo control } & Controlado & 0 & 0 & 0 & 0 & 0.250 \\
\hline & $\begin{array}{l}\text { Control } \\
\text { suficiente }\end{array}$ & 30 & 73.2 & 27 & 65.9 & \\
\hline & Mal controlado & 11 & 26.8 & 14 & 34.1 & \\
\hline & Total & 41 & 100.0 & 41 & 100.0 & \\
\hline
\end{tabular}

En la tabla 3 se muestra que existe diferencia significativa entre la primera y la segunda medición en la medición del IMC antes y después de aplicar el programa $(p<0.5)$ en el $G E$, pasando de tener $64.7 \%$ pacientes con sobrepeso a $49 \%$ después de aplicar el programa de ejercicios. Por otro lado, el GC no mostró una diferencia significativa en relación con su IMC. 
Efectos del programa "Adultos mayores activos y saludables" sobre los niveles de hemoglobina glicosilada y estado antropométrico en pacientes con DM2 mayores de 59 años usuarios del Centro Médico ESSalud Ayaviri, 2018

Tabla 3

Comparación del IMC antes y después de la intervención.

\begin{tabular}{|c|c|c|c|c|c|c|}
\hline \multirow[t]{2}{*}{ Grupo } & \multirow[t]{2}{*}{ IMC } & \multicolumn{2}{|c|}{ Pre test } & \multicolumn{2}{|c|}{ Pos test } & \multirow[t]{2}{*}{$\mathbf{p}^{*}$} \\
\hline & & $\mathbf{n}$ & $\%$ & $\mathbf{n}$ & $\%$ & \\
\hline \multirow{4}{*}{$\begin{array}{l}\text { Grupo de } \\
\text { estudio }\end{array}$} & Normal & 17 & 33.3 & 25 & 49.0 & \multirow[t]{4}{*}{0.011} \\
\hline & Sobrepeso & 33 & 64.7 & 25 & 49.0 & \\
\hline & Obesidad & 1 & 2.0 & 1 & 2.0 & \\
\hline & Total & 51 & 100.0 & 51 & 100.0 & \\
\hline \multirow[t]{4}{*}{ Grupo control } & Normal & 5 & 12.2 & 7 & 17.1 & \multirow[t]{4}{*}{0.157} \\
\hline & Sobrepeso & 35 & 85.4 & 33 & 80.5 & \\
\hline & Obesidad & 1 & 2.4 & 1 & 2.4 & \\
\hline & Total & 41 & 100.0 & 41 & 100.0 & \\
\hline
\end{tabular}

En la tabla 4 se aprecia que existe una diferencia estadísticamente significativa entre la primera y la segunda medición del ICC $(p<0.5)$ en el GE. En el pre test el $51 \%$ estuvo sin complicaciones, después del programa el grupo se incrementó a 70.6\%. Así mismo, el GC no presentó significancia alguna en la reducción de los valores de ICC.

Tabla 4

Comparación del ICC antes y después de la intervención.

\begin{tabular}{|c|c|c|c|c|c|c|}
\hline \multirow[t]{2}{*}{ Grupo } & \multirow[t]{2}{*}{ ICC } & \multicolumn{2}{|c|}{ Pre test } & \multicolumn{2}{|c|}{ Pos test } & \multirow[t]{2}{*}{$\mathbf{p}^{*}$} \\
\hline & & $n$ & $\%$ & $\mathbf{N}$ & $\%$ & \\
\hline \multirow[t]{3}{*}{ Grupo de estudio } & Sin complicaciones & 26 & 51.0 & 36 & 70.6 & \multirow[t]{3}{*}{0.031} \\
\hline & Con complicaciones & 25 & 49.0 & 15 & 29.4 & \\
\hline & Total & 51 & 100.0 & 51 & 100.0 & \\
\hline \multirow[t]{3}{*}{ Grupo control } & Sin complicaciones & 16 & 39.0 & 20 & 48.8 & \multirow[t]{3}{*}{0.219} \\
\hline & Con complicaciones & 25 & 61.0 & 21 & 51.2 & \\
\hline & Total & 41 & 100.0 & 41 & 100.0 & \\
\hline
\end{tabular}

En la tabla 5 se observa que hay significancia estadística en cuanto al estado nutricional de los pacientes con DM2 del GE $(p<0.5)$, mientras que en el GC no se encontró una diferencia significativa $(p>0.5)$.

Tabla 5

Comparación del MNA antes y después de la intervención.

\begin{tabular}{|c|c|c|c|c|c|c|}
\hline \multirow[t]{2}{*}{ Grupo } & \multirow[t]{2}{*}{ MNA } & \multicolumn{2}{|c|}{ Pre test } & \multicolumn{2}{|c|}{ Pos test } & \multirow[t]{2}{*}{$\mathbf{p}^{*}$} \\
\hline & & $\mathbf{N}$ & $\%$ & $n$ & $\%$ & \\
\hline \multirow[t]{4}{*}{ Grupo de estudio } & Normal & 20 & 39.2 & 48 & 94.1 & \multirow[t]{4}{*}{0.001} \\
\hline & Riesgo de malnutrición & 30 & 58.8 & 3 & 5.9 & \\
\hline & Malnutrición & 1 & 2.0 & 0 & 0 & \\
\hline & Total & 51 & 100.0 & 51 & 100.0 & \\
\hline \multirow[t]{4}{*}{ Grupo control } & Normal & 2 & 4.9 & 2 & 4.9 & \multirow[t]{4}{*}{0.206} \\
\hline & Riesgo de malnutrición & 38 & 92.7 & 34 & 82.9 & \\
\hline & Malnutrición & 1 & 2.4 & 5 & 12.2 & \\
\hline & Total & 41 & 100.0 & 41 & 100.0 & \\
\hline
\end{tabular}




\section{DISCUSIÓN}

En la tabla $\mathrm{N}^{\circ} 2$ muestran que no existe significancia estadística, ya que los valores de HbA1C no disminuyeron lo suficiente, resultados que se contradicen con los de Praga (2016) en la investigación titulada "Ejercicio de alta intensidad VS ejercicio de moderada intensidad en pacientes con Diabetes Mellitus 2", en el que, si existió diferencia significativa de $1 \%$ en la disminución de la HbA1c, después de aplicar un programa de ejercicio físico que duró también 12 semanas como el nuestro. Sin embargo, en el estudio de Abarquero (2015), titulado "Beneficios del ejercicio físico en la Diabetes mellitus tipo 2" muestra que los ejercicios deben durar por lo menos 24 semanas para que haya una mejoría significativa en los niveles de HbA1c., lo que consideramos fue el motivo por lo que no se obtuvo un efecto estadístico significativo en los resultados.

En la tabla $\mathrm{N}^{\circ} 3$ se encontró una diferencia estadísticamente significativa en la reducción de los valores del IMC, es decir, sobre el estado nutricional de los pacientes con DM2 del GE; al contrario, el GC no se encontró significancia estadística. En relación a nuestro estudio, existe un incremento en el riesgo de desarrollar diabetes a medida que el índice de masa corporal aumenta, ya sea que la persona venga o no con antecedentes de diabetes en su familia. Alrededor del $90 \%$ de las personas con DM2 tienen un alto IMC oscilando entre $27.0 \mathrm{~kg} / \mathrm{m} 2$ (Bautista y Sanchez, 2013). Por tanto, este contexto concuerda con el programa que desarrollamos ya que los valores de IMC oscilaron entre 27 a $32 \mathrm{~kg} / \mathrm{m} 2$ en adultos mayores con DM2 que se sometieron al estudio. Por otro lado Bautista y Sanchez (2013) en su trabajo de investigación titulado "Asociación del Indice de masa corporal con la hemoglobina glicosilada en adultos de 20 a 65 años de edad, con diabetes tipo 2 en la clínica de obesidad y diabetes de Toluca" muestra que el IMC no está relacionado con el porcentaje de hbA1C de un paciente adulto con Diabetes tipo 2, ya que puede depender de otros factores (alteración genética o química de la hemoglobina puedes incrementarla o disminuirla) y no directamente del peso corporal (Bautista y Sanchez, 2013); que en comparación con nuestro estudio los resultados mostraron que el IMC disminuyo en las personas que tenían sobrepeso, después de haber cumplido con las 12 semanas de ejercicios, y esas mismas personas disminuyeron a su vez el porcentaje de hb1Ac al igual que las personas en estado normal, por lo que la práctica constante de ejercicio está relacionada a la disminución de $\mathrm{HbA} 1 \mathrm{c}$ según nuestros resultados.

También se puede observar que la disminución de peso se redujo solo en personas con sobrepeso a diferencia de las personas obesas. Ya que Gonzalo (2015) en su investigación titulada “Efecto de diferentes programas de entrenamiento en sujetos con sobrepeso sedentarios" refiere que no se puede generalizar a sujetos con sobrepeso y obesidad ya que su metabolismo y sistema endocrino funcionan de forma diferente, y hace hincapié que los resultados de mejora en cuanto a IMC también se ve afectado por el tiempo del programa de ejercicio mayor a 12 semanas (Gonzalo, 2015) ,ya que nuestro estudio duró 12 semanas podemos ver que los participantes con diagnóstico de obesidad mantuvieron su peso.

Asimismo, menciona que un $44 \%$ de la población con obesidad dijeron que la salud y la nutrición estaban entre las 3 consideraciones principales para comprar comida sin embargo el $62 \%$ de los encuestados obesos admitieron que sabían que deberían de comer en forma saludable, pero que no lo hacían y era más probable que ignoraran las etiquetas nutricionales y optaran por snacks y refrigerios

Observando estos resultados otros factores a tener en cuenta en la disminución de peso en las personas con obesidad, es la conducta alimentaria y problemas psicológicos que hacen que a estos sujetos les sea más difícil dejar sus hábitos pasados (Mozes, 2018). Considerando estos factores, en nuestro estudio no se siguió un plan de alimentación estricto, propuesto para estas personas; ya que nos basamos solo observar la influencia que tiene el ejercicio combinado en este grupo de adultos mayores con DM2 para disminuir el porcentaje de $\mathrm{HbA} 1 \mathrm{c}$.

A su vez la tabla $\mathrm{N}^{\circ} 4$, nos muestra que existe una relación entrela reducción del perímetroabdominal y el ejercicio combinado. Arabia, et al, (2012),en su estudio llamado "Ejercicio en el tratamiento de la diabetes mellitus 2" nos dice que el ejercicio aeróbico y el de fuerza mejoran el control glucémico, reducen el riesgo cardiovascular y contribuyen a la perdida y mantenimiento de peso (Arabia, et al, 2012).apoyando nuestros resultados ya que el porcentaje de ICC disminuyó de $49 \%$ con personas en riesgo cardiovascular a un 29.4\%; así como Parra, et al (2015) en su 
estudio "Evaluación de un programa de ejercicio supervisado en pacientes sedentarios mayores de 65 años con diabetes mellitus tipo 2" demuestra la relación entre la reducción de circunferencia abdominal y porcentaje de grasa con los valores de Hb1Ac y en pacientes con DM2 está basado en la producción de adipocinas que intervienen en el metabolismo de la glucosa es mayor en la grasa abdominal que la grasa corporal, concluyendo que la reducción del porcentaje de grasa influye en un $40 \%$ de los cambios en hemoglobina glucosilada y la reducción de grasa corporal influye en un $25 \%$ de los cambios en hemoglobina glucosilada (Parra, 2015).

Sin embargo, nuestro estudio no muestra una relación fuerte entre la reducción del ICC y la disminución de $\mathrm{Hb} 1 \mathrm{Ac}$, ya que no necesariamente disminuyo la $\mathrm{HbA} 1 \mathrm{c}$ a la par con el porcentaje de grasa o ICC, al igual que el IMC.

\section{CONCLUSIÓN}

No existe efecto estadísticamente significativo entre el programa de ejercicios "Adultos mayores activos y saludables" ( $p>$.05) y la disminución de $\mathrm{Hb} 1 \mathrm{Ac}$ en adultos mayores con DM2.

Sin embargo, en los resultados de laboratorio existe una reducción significativa en el porcentaje de $\mathrm{HbA} 1 \mathrm{c}$.

Si existe efecto entre el programa de ejercicios "Adultos mayores activos y saludables" y el estado antropométrico en adultos mayores con DM2.
No existe efecto entre el programa de ejercicios "Adultos mayores activos y saludables" a través del ICC sobre los niveles de Hb1Ac.

Considerando que la ciudad donde se realizó este estudio tiene menos incidencia de DM2, se recomienda la aplicación de este programa de ejercicios en la región Costa de nuestro país ya que durante la investigación esta resultó siendo la que mayor índice de DM2 tenía.

Se recomienda la realización de un programa de ejercicio combinado en pacientes con DM2 de por lo menos 24 semanas con el fin de comprobar la efectividad a largo plazo. Se insta a la evaluación de mayores valores antropométricos como el porcentaje de grasa para ver la relación que tiene este con la $\mathrm{Hb} 1 \mathrm{Ac}$.

\section{Declaración de financiamiento y de conflictos de interés:}

El estudio fue financiado por los autores, quienes declaran no tener conflictos de interés.

\section{Correspondencia}

Patthy Milagros Condori Choquepata

Correo electrónico:

patthy2016@hotmail.com

Tirsa Abigail Mauricio Navarro

Correo electrónico:

tichamauricio90@gmail.com 


\section{REFERENCIAS BIBLIOGRÁFICAS}

Abarquero I (2015). Beneficios del ejercicio en la Diabetes Mellitus tipo 2; 26p.

Arabia M, Suárez R, Tróchez M. (2012).El ejercicio en el tratamiento de la Diabetes mellitus tipo 2. Revista Argentina de endocrinología y metabolismo, 48(4): 203-212.

Bautista K, Sánchez L. (2013). Asociación del Indice de Masa Corporal con la hemoglobina glicosilada(hbA1c) en adultos de 20 a 65 años de edad, con Diabetes tipo 2 en la Clínica de Obesidad y Diabetes, de Toluca, estado de Mexico; 36p.

Encuesta demográfica y de salud familiar (ENDES) (2012), instituto nacional de estadística e informática. Recuperado de http://proyectos.inei. gob.pe/web/biblioineipub/bancopub/est/lib1075/ Libro.pdf

Fraga P (2016) Ejercicio de alta intensidad VS ejercicio de moderada-baja intensidad en pacientes con Diabetes Mellitus tipo 2. España; pp43.

Fernandes J, Ogurtsova K, Linnenkamp U, Guariguata L, Seuring T, Zhang P, (2016). IDF Diabetes Atlas estimates of 2014 global health expenditures on diabetes. Diabetes research and clinical Prac.; 117: 48-54.

Gonsáles M. (2016).Actividad física y programa de ejercicio en el adulto mayor: necesidades no aplicadas en su verdadera dimensión. Rev. Cuerpo. Méd. HNAA.

Gonzalo G. (2015).Efecto de diferentes programas de entrenamiento en sujetos con sobrepeso y sedentarios. Universidad Nacional de la Plata; $74 \mathrm{p}$.

International Diabetes Federation (2017). Atlas de la Diabetes de la FID.; 8va ed. 150p.
Mamani D (2016). Depresión, Ansiedad y estado nutricional del Adulto mayor albergado en centros de atención residencial de la provincia de PunoChucuito; 72p.

Ministerio de Salud (2016). Guía de Práctica para el diagnóstico, Tratamiento y Control de la Diabetes Mellitus Tipo 2 en el Primer Nivel de Atención. $63 p$.

Mozes A. (2018). El motivo de que para los obesos bajar de peso resulte tan difícil. Recuperado de: https://consumer.healthday.com/espanol/ vitamins-and-nutrition- information-27/obesityhealth-news-505/el-motivo-de-que-para-losobesos-bajar-de-peso-resulte-tan-dif-iacutecil-735072.html

Paredes A. (2017). Diabetes mellitus y enfermedad crónica en el HRMNB. Puno;. 68p.

Parra J, Moreno M (2015). Evaluación de un programa de ejercicio físico supervisado en pacientes sedentarios mayores de 65 años con diabetes mellitus tipo 2. Aten Primaria. España.

Seclén S. (2015). Diabetes Mellitus en el Perú: hacia dónde vamos. Rev Med Hered.26: 3-4.

Villena J. (2016). Epidemiología de la Diabetes Mellitus en el Perú. Diagnóstico; 55(4): 173-181.

Recibido: $16 / 10 / 18$

Aceptado: 10/01/19 